\title{
Bioética, imigração e assistência à saúde: tensões e convergências sobre o direito humano à saúde no Brasil na integração regional dos países
}

\author{
Bioethics, immigration and health care: tensions and \\ convergences on the human right to health in Brazil in the regional \\ integration of the countries
}

Katia Guerra1, Miriam Ventura²

\begin{abstract}
Resumo
Introdução: Os fluxos imigratórios são um fenômeno mundial e o Brasil não fica distante desse processo. Embora a migração não repercuta necessariamente como uma ameaça à saúde, ela pode aumentar a vulnerabilidade dos sujeitos, tornando imprescindíveis políticas públicas que garantam o direito à saúde. Este artigo teve como objetivo analisar os aspectos bioéticos relacionados ao cumprimento do direito humano ao acesso aos serviços de saúde pelos imigrantes no Brasil. Método: Busca eletrônica na base de dados SciELO e revistas de bioética, direitos humanos e saúde coletiva, sem restrição de idioma e ano de publicação. Resultado: Foram identificados 16 trabalhos categorizados em imigrantes do MERCOSUL e outras origens, e imigrantes situados em região de fronteira. O Brasil vem procurando respeitar o direito humano à saúde aos imigrantes por meio de novas formas de atuação em saúde, com ações e estratégias específicas, processos de trabalhos mais expandidos e flexíveis; mas existiram enfrentamentos éticos no tocante à equidade, universalidade e não discriminação. Conclusão: A criação e o aperfeiçoamento constante de normas e práticas são necessários para que se alcance a responsabilidade ética com esta população e o acesso à saúde no Brasil se consolide como um direito humano universal, principalmente nas regiões de fronteira.
\end{abstract}

Palavras-chave: imigração; direito humano à saúde; bioética; Brasil; MERCOSUL.

\begin{abstract}
Introduction: Migratory flows are a global phenomenon, a process in which Brazil is involved. Although migration doesn't necessarily have repercussions as a health threat, it can increase the vulnerability of individuals, making public policies that guarantee the right to health care essential. This article aims to analyze bioethical issues related to the fulfillment of the human right to access health services for immigrants in Brazil. Method: An electronic search in the SciELO database and bioethics, human rights and public health journals, without restriction for language or year of publication. Result: The present literature review identified 16 studies categorized according to MERCOSUR immigrants and other sources, and immigrants located on the border region. Brazil is seeking to respect the human right to health for immigrants through new forms of health care related activities, with specific measures and strategies, and more expanded and flexible work processes; but there were ethical clashes with regard to equity, universality and non-discrimination. Conclusion: It's still necessary to create and constantly improve policies and practices for achieving ethical responsibility to this population and the access for health care in Brazil to be consolidated as a universal human right, especially in border regions.
\end{abstract}

Keywords: immigration; human right to health; bioethics; Brazil; MERCOSUR.

${ }^{1}$ Subsecretaria de Regulação, Controle e Avaliação, Contratualização e Auditoria, Secretaria Municipal de Saúde do Rio de Janeiro (SMSRJ) - Rio de Janeiro (RJ), Brasil.

22Área de Ciências Humanas e Sociais, Instituto de Estudos em Saúde Coletiva (IESC), Universidade Federal do Rio de Janeiro (UFRJ) - Rio de Janeiro (RJ), Brasil. Trabalho realizado no Programa de Pós-graduação em bioética, ética aplicada e saúde coletiva (PPGBIOS), Instituto de Estudos em Saúde Coletiva (IESC) - Rio de Janeiro (RJ), Brasil.

Endereço para correspondência: Katia Guerra - Coordenadoria Geral de Controle e Avaliação (CGCA), Subsecretaria de Regulação (SUBREG), Secretaria Municipal de Saúde do Rio de Janeiro (SMSRJ), Rua Afonso Cavalcanti, 455, sala 812 - Cidade Nova - CEP: 20211-110 - Rio de Janeiro (RJ), Brasil -

Email:kcmguerra@gmail.com

Fonte de financiamento: nenhuma.

Conflito de interesses: nada a declarar.

Cad. Saúde Colet., 2017, Rio de Janeiro, 25 (1): 123-129 


\section{INTRODUÇÃO}

Os fluxos imigratórios são um fenômeno mundial em ritmo crescente, tornando relevantes as discussões sobre políticas públicas para o acesso à saúde de imigrantes. $\mathrm{O}$ Brasil não fica distante deste processo; o país está entre as 10 maiores economias mundiais, sediou a Copa do Mundo em 2014 e as Olimpíadas em 2016, motivos que o tornam atrativo para a imigração em razão do maior número de investimentos e da procura de mão de obra.

Os processos de integração regional dos países iniciados pela construção da União Europeia também contribuem na imigração. No Brasil, já podem ser identificadas influências e repercussões no setor saúde, em especial, relacionadas ao aumento do livre comércio representado pela participação do país no Mercado Comum do Sul (MERCOSUL) ${ }^{1}$.

Outro fator que adquire elevada importância é a larga faixa fronteiriça brasileira. São 569 municípios em $15.719 \mathrm{~km}$ e a procura por atendimento de cidadãos de outros estados membros do MERCOSUL já é uma realidade nos municípios fronteiriços brasileiros.

Embora a migração não repercuta necessariamente como uma ameaça à saúde, ela pode aumentar a vulnerabilidade dos sujeitos, considerando as diferentes características da população migrante e nativa, e dos próprios sistemas de saúde vigentes nos países. Esses aspectos individuais, programáticos e sociais da vulnerabilidade devem ser pensados na elaboração e implementação de políticas de saúde ${ }^{2}$.

Pelo fato da possibilidade de uma parcela de imigrantes serem afetados negativamente pela experiência migratória é que se tornam imprescindíveis normas e práticas institucionais que visualizem essas vulnerabilidades $\mathrm{e}$ atendam às necessidades específicas desse segmento, de forma a garantir o direito ao acesso à saúde deste grupo.

O direito à saúde está presente na Declaração Universal dos Direitos Humanos. A saúde é definida como uma condição para uma vida digna e indispensável para a usufruição de outros direitos como a igualdade, a educação, a liberdade, componentes integrais para ter uma vida saudável ${ }^{3}$.

Nesse sentido, as restrições ao acesso aos bens e serviços de saúde merecem especial cuidado nas suas justificativas éticas, considerando que podem representar grave violação aos direitos humanos.

A abordagem teórica metodológica adotada para o estudo é a da Bioética e buscará explorar as articulações desta com a Saúde Coletiva.

A Bioética propõe: analisar e criticar racionalmente o agir humano e oferecer soluções normativas que possam ser positivas do ponto de vista do bem-estar e da saúde dos seres vivos.

A Bioética difere da ética tradicional e passa a adotar características epistemológicas multidisciplinares, interdisciplinares e transdisciplinares. Como aponta Junges e Zoboli ${ }^{4}$, a Bioética, como campo de saber específico, no plano do discurso científico e das práticas sociais, pode potencializar as lentes para a análise da realidade material das ações.

Nesse sentido, considerando os conflitos éticos presentes nas restrições impostas aos imigrantes para o acesso à saúde e a complexidade e desafios para os sistemas de saúde dos fluxos migratórios, o estudo pretende analisar como o Brasil está assegurando este direito e enfrentando este desafio na atualidade, e indagar sobre as possíveis dificuldades e limitações na utilização dos serviços de saúde brasileiros pelos imigrantes.

\section{MÉTODOS}

Este artigo é o resultado de uma revisão de literatura para analisar aspectos bioéticos relacionados ao cumprimento do direito humano ao acesso aos serviços de saúde pelos imigrantes no Brasil, que emergem ou são apontados nos estudos sobre o tema.

Para tanto, foi realizada busca na base de dados eletrônica SciELO, em Revistas especializadas em bioética, direitos humanos, saúde coletiva e pública, Google acadêmico, incluindo publicações, teses e dissertações em banco da CAPES-CNPq.

Não houve critérios de restrições de idioma e nem especificado o prazo para período de busca, visando ampliar ao máximo a pesquisa bibliográfica.

Foram usados termos isolados e combinados para identificar trabalhos relacionados com o tema: saúde, imigrante, MERCOSUL, direitos humanos, bioética e saúde pública. $\mathrm{Na}$ articulação das palavras, foi adotada a expressão booleana "AND" que permite a inserção de duas ou mais palavras. A revisão teórica pretendeu analisar as abordagens sobre a problemática do direito humano à saúde do imigrante e de pesquisas no contexto brasileiro sobre a temática.

Os critérios para inclusão da busca bibliográfica foram: pesquisas empíricas sobre o acesso à saúde de imigrantes, estudos teóricos e reflexões éticas, bioéticas e de direitos humanos sobre a problemática.

Foram excluídos trabalhos duplicados, de imigrantes refugiados e solicitantes de asilo.

\section{RESULTADOS}

Inicialmente foram encontrados 34 trabalhos e, após leitura criteriosa dos seus resumos, foram selecionados 22 estudos. Em seguida, foi feita leitura flutuante de todo o corpo destes trabalhos para identificar quais realmente abordavam a temática, chegando a uma amostra de 16 trabalhos que atendiam aos objetivos e critérios de inclusão e exclusão. Posteriormente, foi feita a leitura integral do material e sua caracterização. 
Dos artigos selecionados, 2 são em inglês e 2 em espanhol. Eles foram divididos em 2 categorias: imigrantes do MERCOSUL e de outras origens; e imigrantes situados em região de fronteira.

\section{Imigrantes no acesso aos serviços de saúde brasileiros}

Embora o número de imigrantes venha crescendo no Brasil, há poucos trabalhos que investigam o tema do acesso aos serviços de saúde. Foram encontrados apenas 12 estudos empíricos com os imigrantes bolivianos, japoneses e coreanos na cidade de São Paulo e 4 nas regiões de fronteira com Argentina, Paraguai e Uruguai.

A região metropolitana de São Paulo é apontada como a maior receptora de imigrantes do país, sendo a nacionalidade boliviana a mais expressiva ${ }^{5}$.

Em um dos primeiros trabalhos, foi apontada a dificuldade dos imigrantes bolivianos que trabalhavam nas oficinas de costura no acesso aos serviços de saúde em razão de não possuírem documentação, não falar a língua local e a falta da qualidade no atendimento ${ }^{6}$.

Carneiro e Silveira ${ }^{7}$ verificaram maior prevalência em situações de violência doméstica e laboral de bolivianas em relação a outras usuárias do Centro de Saúde pesquisado. Para contornar esta situação, foi criado um atendimento com abordagem transdisciplinar, envolvendo equipe de saúde, advogados e cientistas sociais.

Já Xavier ${ }^{8}$ ressaltou o papel da Estratégia Saúde da Família (ESF) na criação de vínculo com as unidades de saúde e no processo de integração à sociedade dos bolivianos em São Paulo. Ainda relata que os imigrantes adquiriram o cartão do Sistema Único de Saúde (SUS) como seu primeiro documento brasileiro.

Silva ${ }^{9}$ também fez uma análise positiva a respeito da interação entre os profissionais da ESF, em especial os agentes de saúde, e os bolivianos. Comenta que não houve relatos de dificuldade no acesso aos serviços de saúde, mas que, inicialmente, tanto os profissionais como outros usuários apresentaram resistências, com atitudes de hostilidade, rejeição e intolerância.

Assim como Waldman ${ }^{10}$ também constatou que apesar de os bolivianos do seu estudo já terem tido atendimento no SUS, aconteceram pequenas situações de desrespeito dos profissionais da unidade, como falarem alto com eles e empurrá-los.

Melo e Campinas ${ }^{5}$ demostraram grande incidência de tuberculose entre a população boliviana associada às condições insalubres das oficinas de costura, jornadas de trabalho exaustivas, abandonos de tratamento, além do aumento do índice do vírus da imunodeficiência humana (HIV). Mas também destacaram a importância do vínculo obtido pela ESF contribuindo para a eficácia do tratamento.

A cidade de São Paulo começou a criar ações específicas para os bolivianos em 2003 em decorrência do aumento dos seus índices de HIV e tuberculose. Dentre as ações estão: contrato de bolivianos para atuarem como agentes de saúde, informativos sobre prevenção de HIV e tuberculose em espanhol, e prestação de cursos de espanhol para os profissionais das unidades?.

Martes e Faleiros ${ }^{11}$ realizaram entrevistas com bolivianos recém-chegados a São Paulo e foram citados casos de discriminação e problemas em compreender as orientações médicas. Mas, no conjunto, os entrevistados demonstraram satisfação com os serviços disponibilizados e a qualidade do atendimento no SUS, principalmente pela gratuidade dos serviços e de vários medicamentos.

O número maior de unidades de saúde; a imunização; e a realização de exames e partos foram elementos pontuados como muito positivos e referenciados em comparação aos encontrados na Bolívia. Contam que lá a maioria dos partos é natural e ocorre na própria casa em razão de ser um procedimento pago, o que acaba resultando em alto índice de mortalidade materna boliviana.

Os depoimentos valorizaram o abrandamento da dor física por meio de acesso a medicamentos, tratamentos e partos, incluído a cesariana ${ }^{11}$.

A ESF também foi referenciada positivamente como um facilitador ao processo de integração e por realizar ações diretamente no domicílio dos entrevistados. Embora possuíssem o cartão SUS, isto não era uma condição para atendimento, sendo possível apenas com a carteira de identidade boliviana. Isto evidencia a não restrição do acesso pelo status legal de estar regularizado no país, reforçando o caráter universal e gratuito do SUS ${ }^{11}$.

Outros fatores apontados como facilitadores na promoção do acesso estão: as redes familiares, os agentes comunitários e os proprietários de oficinas. Foram poucas as falas de discriminação ocorridas geralmente no setor de cadastro das unidades e não apenas pela nacionalidade, mas pela origem indígena ${ }^{11}$.

Rufino e Amorim ${ }^{12}$ pesquisaram algumas das práticas de gestão pública para o atendimento de imigrantes em São Paulo. Constataram que a ESF é mais efetiva que as unidades básicas tradicionais pela característica de visitarem os pacientes em suas casas e junto das famílias de forma que promovem maior interação, contribuindo para um atendimento mais voltado às suas necessidades.

Embora tenham tido iniciativas como a contratação de profissionais e de informativos na língua espanhola, estas não se mostraram suficientes e as ações não foram institucionalizadas.

Apontam a necessidade de formulação de novas políticas públicas e de aperfeiçoamento das já existentes, já que, tanto os gestores como os profissionais de saúde, não estão preparados para atender à especificidade da população migrante.

Aguiar e Mota ${ }^{13}$ verificaram que os bolivianos mudam constantemente de moradia devido à busca por melhores colocações em outras oficinas de costura, sendo necessário 
flexibilizar as normativas da ESF para que não se comprometa o vínculo já adquirido e nem a continuidade do tratamento, em especial o pré-natal e o combate à tuberculose. Entre as adaptações está a de manter o cadastro na unidade mesmo com a mudança para outra área.

Também foram feitas reuniões de sensibilização com os donos das oficinas para permitir que os imigrantes interrompessem o trabalho para irem às unidades e possibilitassem a entrada dos agentes nas oficinas para realizarem as visitas domiciliares.

Uma das principais queixas dos bolivianos é a dor muscular devido às longas jornadas de trabalho, o que promoveu o início da prestação de exercícios de alongamento nas próprias oficinas.

Os profissionais também fazem participações nas rádios comunitárias bolivianas, passando informações sobre saúde e comparecendo a feiras frequentadas pelos imigrantes. Estas estratégias de comunicação visam melhorar a interação dos imigrantes com os serviços e sua integração à sociedade

Goldberg e Silveira ${ }^{14}$ revelaram que imigrantes bolivianos enfrentaram obstáculos em hospitais públicos em Buenos Aires e São Paulo e, não obstante tenham verificado um aumento do acesso e da cobertura de saúde, não foram estabelecidas políticas intersetoriais e serviços de saúde que efetivamente realizem a inclusão deste grupo.

Chubaci e Merighi ${ }^{15}$ realizaram estudo com imigrantes japoneses em um hospital do SUS em São Paulo e concluíram que a dificuldade com a língua portuguesa promove reflexos negativos na compreensão da doença, no tratamento e no trato com os profissionais de saúde. Estas condições provocam um sentimento de insegurança tanto para explicarem o que sentem quanto para compreenderem o que os profissionais dizem.

A barreira do idioma ainda é agravada quando a doença é mais grave, devido à maior utilização pelos profissionais de termos técnicos para esclarecê-la, além da dificuldade de compreensão das instruções durante os procedimentos que dependem da colaboração dos pacientes para serem realizados.

Em razão do problema de comunicação, relatam não conversarem com ninguém, o que os leva ao sofrimento pela angústia e tristeza de não poderem expressar em detalhes o que sentem. Alguns pensam que se a equipe médica escrevesse em português lhes facilitaria a compreensão.

O estudo revelou a importância que a comunicação, tanto verbal como não verbal, tem no relacionamento interpessoal em processos de hospitalização, e a necessidade de que os profissionais de saúde tenham sensibilidade para compreender o teor das mensagens enviadas pelos pacientes, de forma a poder prestar uma assistência mais eficiente e adequada às suas necessidades.

Também mostra que a disponibilidade de serviços de saúde não é suficiente e que outras ações devem ser pensadas para que promovam outras dimensões do acesso para o alcance do bem-estar das pessoas.
Kang et al. ${ }^{16}$ verificaram que os imigrantes coreanos em São Paulo apresentam mais transtornos psiquiátricos do que a população coreana na terra natal. Este resultado mostra que programas de saúde mental devem dar mais atenção a este e a outros grupos de imigrantes para que tenham uma integração mais saudável, já que a literatura mostra que transtornos psiquiátricos são comuns entre imigrantes.

\section{Zonas de fronteira brasileira}

As experiências em zonas de fronteira revelam a complexidade da garantia do acesso à saúde aos imigrantes. Nestas zonas, podem ocorrer desigualdades assistenciais e discriminatórias entre pessoas de uma mesma região, porém de países diferentes.

Uma das ações do governo brasileiro voltada ao acesso dos imigrantes que residem nestas regiões foi a criação, em 2005, do Programa Sistema Integrado de Saúde nas fronteiras (SIS fronteiras). Foi desenvolvido com a finalidade de organizar o sistema nos municípios fronteiriços brasileiros com Argentina, Paraguai e Uruguai.

Nogueira e Fagundes ${ }^{17}$ avaliaram o SIS fronteiras entre 2009 e 2011 e identificaram dificuldades no campo da gestão, principalmente, pela descontinuidade administrativa, ausência de assessoria federal e estadual; e mudança da equipe ministerial responsável pelo programa.

Estes fatores não contribuíram para que os objetivos iniciais fossem atingidos e, a partir de 2010, o Ministério da Saúde não se pronunciou mais sobre o andamento do programa.

Jimenez e Nogueira ${ }^{18}$ estudaram a região da Tríplice Fronteira, uma área formada por 3 cidades, pertencentes cada uma ao Brasil, ao Paraguai e a Argentina, com um total de 700 mil habitantes. A região apresenta uma situação de desigualdade no acesso à saúde devido à assimetria dos sistemas de saúde nacionais com diferentes proteções sanitárias. A população dos países vizinhos busca atendimento no Brasil pelo fato de o SUS ser universal e gratuito, diferentemente do caso do Paraguai e da Argentina.

Segundo os autores, na cidade brasileira, a população vizinha é atendida em casos de urgência e emergência; e com possibilidade de acesso aos outros níveis de atenção por meio de uma autorização da prefeitura local.

Apesar da existência do interesse em estabelecer acordos com as autoridades sanitárias vizinhas, não há fluxos estruturados formais dos serviços de saúde entre as 3 cidades. Pequenas ações conjuntas de vigilância e registros epidemiológicos estão sendo promovidos pela Comissão de Saúde do Comitê da Fronteira e pelo grupo de trabalho (GT) para a Integração das Ações em Saúde da Hidroelétrica Itaipu Binacional.

Para os autores, no bloco do MERCOSUL, as iniciativas legais e formais ficam paralisadas no nível dos governos nacionais sem alcançar os municipais, e sem provocar mudanças nas desigualdades territoriais fronteiriças. 
Giovanella et al. ${ }^{19}$ realizaram inquéritos com secretários municipais de saúde de 69 municípios brasileiros fronteiriços com Argentina, Paraguai e Uruguai. Concluíram que a procura por serviços do SUS é maior por brasileiros residentes nos municípios estrangeiros do que pelos estrangeiros. Apesar de todos os entrevistados afirmarem já ter prestado algum tipo de atendimento a estrangeiros, foram encontradas barreiras ao acesso tanto para brasileiros não residentes como para estrangeiros, como a necessidade de comprovação de residência brasileira e carência de 30 dias para atendimento a novos moradores.

Embora os secretários relatem um alto atendimento a estrangeiros, a maioria não os registra como tal. Esta falha no registro foi apontada como a principal dificuldade para a continuidade do atendimento e referências para outros níveis de atenção, tanto para as cidades brasileiras quanto para as estrangeiras. Relataram ainda a não existência de acordos formalmente estabelecidos, mas apenas iniciativas locais de cooperação, e em somente metade dos municípios participantes da pesquisa.

Citaram como formas de cooperação o GT da Itaipu Binacional na fronteira com o Paraguai e a Comissão de Saúde da Fronteira com Uruguai. Afirmaram que a formalização de cooperação nas fronteiras favoreceria a garantia da assistência integral até mesmo para os brasileiros residentes em razão da possibilidade do serviço de saúde mais próximo estar no outro lado da fronteira.

Nogueira et al. ${ }^{20}$ realizaram estudo com gestores e profissionais de saúde de 26 municípios brasileiros fronteiriços com Argentina, Paraguai e Uruguai.

Destacaram dentre os resultados encontrados as diferentes concepções pelos entrevistados do direito à saúde dos estrangeiros, o que pode gerar negativas ao acesso ou apenas o atendimento em casos de urgência e emergência, levando a incertezas quanto à garantia do direito.

As estratégias e as práticas para a inclusão e a oferta dos serviços de saúde aos estrangeiros foram geralmente pontuais, nem sempre direcionadas às necessidades de saúde, sem uma padronização uniforme e não adquiriram visibilidade a ponto de se tornarem institucionalizadas.

Verificaram ainda que os acordos e pactos firmados pelos governos nacionais não tiveram repercussão e nem eram de conhecimento por parte de alguns entrevistados.

Destacaram, como pontos positivos, na busca dos serviços no SUS, a gratuidade do sistema e a qualidade do atendimento; e, como negativos, a ESF, pela adstrição da clientela, restringindo-se a usuários residentes em um território específico e ao cartão SUS, em função da necessidade de comprovante de residência brasileira para seu registro e aquisição.

Foram poucos os aspectos culturais apontados pelos entrevistados que interferiram na interação com os estrangeiros, dentre eles: o alto número de indígenas, o idioma guarani e a crença na medicina natural.

\section{DISCUSSÃO}

A condição de saúde dos imigrantes está entre os pontos mais sensíveis para a concretização da inserção e integração à sociedade. Portanto, é preciso pensar nas consequências negativas que a imigração traz para a saúde e as responsabilidades dos Estados para com a saúde desta população.

Uma das responsabilidades que se ampliam para os blocos de países em processos de integração regional como o Brasil é a assistência à saúde individual, tornando necessária a criação de políticas de inclusão não discriminatórias, estruturadas em bases éticas, para que se obtenham resultados eficazes.

O direito à saúde discutido sob a perspectiva dos direitos humanos, pressupõe o respeito à dignidade humana e o dever dos Estados em garantir condições para o exercício deste direito através de leis e políticas públicas.

Foi possível identificar nos estudos analisados uma defesa da cultura dos direitos humanos e da difusão de princípios éticos relacionados ao acesso universal, sem discriminação e gratuidade da assistência à saúde.

E, dentre estes princípios, sobressaiu-se o da proteção à pessoa como fundamento moral do dever estatal de proteger e garantir acesso à saúde dos imigrantes por meio de ações voltadas às necessidades deste grupo, na perspectiva de ética de responsabilidade e justiça social global.

É importante ressaltar que, embora não exista uma regulamentação específica para o acesso à saúde pelo imigrante no Brasil, os princípios normativos adotados pela Constituição Federal Brasileira e a Lei Federal Orgânica do SUS ${ }^{21}$ não vedam o acesso universal e gratuito dos serviços de saúde aos imigrantes no SUS, estejam eles regularizados ou não, membros ou não do MERCOSUL.

No entanto, em parecer produzido pela Advocacia Geral da União em 2008, os estrangeiros com visto de turista, ou em trânsito, ou que residam nos locais de fronteira com o Brasil, não gozam dos mesmos direitos que os brasileiros, devendo-se celebrar acordos bilaterais/multilaterais que prevejam a assistência terapêutica ${ }^{22}$. Tal exigência pode ocasionar barreiras ao direito humano à saúde.

Em contraste, foram apontados avanços nos processos de integração social dos imigrantes em algumas cidades brasileiras, que têm envolvido a criação de novas formas de atuação em saúde, com ações e estratégias específicas, processos de trabalhos mais expandidos e flexíveis.

Os estudos empíricos apontaram uma valorização de princípios bioéticos na assistência ao imigrante, favoráveis às garantias do direito humano global à saúde. Mas é certo que há ainda um longo caminho a percorrer para superar os obstáculos no cumprimento deste direito.

Embora a legislação brasileira de saúde esteja em concordância com esses preceitos éticos de respeito às garantias assistenciais aos imigrantes, os estudos também revelaram iniquidades no acesso aos serviços de saúde, exigindo enfrentamentos éticos no tocante à equidade, universalidade e não discriminação. 
Destaca-se que as medidas realizadas em São Paulo como a contratação de trabalhadores bolivianos e informativos em espanhol se mostraram isoladas e sem garantias políticas-institucionais. A dificuldade de comunicação pelo não conhecimento do idioma e o comportamento dos profissionais nos serviços de saúde também foram pontuados negativamente com relatos de situações de discriminação.

Os profissionais e os gestores não se mostraram preparados quanto às diferenças socioculturais e os aspectos éticos dos imigrantes, e nem sobre a legislação correspondente à proteção dos direitos dessa população.

Aspectos bioéticos como a não discriminação, a igualdade e o respeito às diferenças e especificidades dos imigrantes merecem especial atenção, considerando os relatos de violação também nos estudos das regiões de fronteira brasileira.

Para a garantia do direito ao acesso integral e universal aos serviços de saúde nestes locais, é recomendável a realização de acordos de cooperação e de atividades conjuntas que estabilizem as políticas e programas, e enfrentem, em conjunto, as dificuldades apresentadas diante da complexidade do processo de integração regional nos serviços de saúde.

A criação de políticas de saúde, como a ESF e o cartão SUS, ao mesmo tempo que se mostraram potencialmente favoráveis ao acolhimento da população migrante, apresentaram aspectos que merecem ajustes para afastar alguns obstáculos identificados nos estudos nas regiões fronteiriças. Assim como a melhoria do registro das informações sobre os imigrantes se mostrou como aspecto importante para a assistência, considerando as fragilidades identificadas nos estudos.

Apesar das dificuldades e limitações apresentadas, o Brasil vem buscando implementar o direito humano à saúde também para os imigrantes. Mas o fato de a legislação brasileira estar em acordo com os preceitos éticos dos direitos humanos não está sendo suficiente para atender às necessidades específicas dos imigrantes residentes e nem os que se encontram em trânsito ou em fronteira.

Desta forma é necessária a busca constante de novas formas de atuação, criação de ações, estratégias e políticas que prevejam a igualdade, a não discriminação e reforcem o cumprimento do direito humano à saúde.

\section{CONCLUSÃO}

Embora o âmbito dos estudos identificados na revisão tenha ficado restrito à cidade de São Paulo e a uma faixa da região de fronteiras, não representando a totalidade dos imigrantes no país, foi possível visualizar questões que merecem ser destacadas.

A criação de novas estratégias, tanto para a incorporação como para a continuidade da assistência aos imigrantes em São Paulo, pretendeu englobar os determinantes sociais e subjetivos da saúde e doença, e se revelou primordial para se alcançar um sistema mais efetivo.

Apesar das dificuldades na garantia de processos assistenciais éticos, o modelo do SUS favorece processos de trabalho mais flexíveis com novas modalidades de acesso e de atuação mais adequada à realidade da região, dentro do contexto socioambiental.

No entanto, as tentativas que obtiveram sucesso precisam ser institucionalizadas para não correrem o risco de se perderem com mudanças de gestão e não se tornarem sustentáveis.

É de suma importância que as políticas de saúde sejam desenvolvidas e aplicadas em conformidade com as necessidades das populações migrantes, e que os profissionais das unidades de saúde sejam devidamente instrumentalizados e preparados para atuar junto a eles.

As normas e princípios internacionais dos direitos humanos são compartilhados pela Bioética ao reconhecerem a necessidade de protegerem a dignidade da pessoa humana e assegurar valores essenciais comuns como a saúde.

$\mathrm{Na}$ análise bioética das práticas assistenciais e políticas relacionadas aos imigrantes no Brasil, o estudo aponta que o país vem procurando respeitar o direito humano à saúde não apenas para os imigrantes provenientes do MERCOSUL mas também para o de outras origens.

Promover a solidariedade entre os estados, bem como entre indivíduos e grupos, com especial atenção aos vulneráveis como os imigrantes, é condizente com o princípio da cooperação internacional dos direitos humanos.

Mas é necessário que o país crie e aperfeiçoe constantemente as normas e práticas para que se alcance a responsabilidade ética com esta população e o acesso à saúde no Brasil se consolide como um direito humano universal principalmente nas regiões de fronteira.

\section{REFERÊNCIAS}

1. Lucchese G. Globalização e regulação sanitária: os rumos da vigilância sanitária no Brasil [tese]. Rio de Janeiro: Fundação Oswaldo Cruz, Escola Nacional de Saúde Pública; 2001.

2. Ayres JRCM, França Jr I, Calazans GJ, Saletti Fo HC. O conceito de vulnerabilidade e as práticas de saúde: novas perspectivas e desafios. In: Czeresnia D, Freitas CM. Promoção da saúde: conceitos, reflexões, tendências. Rio de Janeiro: FIOCRUZ; 2003. p. 117-39.

3. Vanderplaat M. Direitos humanos: uma perspectiva para a saúde pública. Revista Saúde e Direitos Humanos. 2004;1(1):27-34.

4. Junges JR, Zoboli ELCP. Bioética e saúde coletiva: convergências epistemológicas. Cien Saude Colet. 2012;17(4):1049-60. PMid:22534858. http://dx.doi.org/10.1590/S1413-81232012000400026.

5. Melo R, Campinas LLSL. Multiculturalidade e morbidade referida por imigrantes bolivianos na Estratégia Saúde da Família. O Mundo da Saúde. 2010;34(1):25-35.

6. Silva SA. Costurando sonhos: trajetória de um grupo de imigrantes bolivianos em São Paulo. São Paulo: Paulinas; 1997. 
7. Carneiro Jr JN, Silveira C. Organização das práticas de atenção primária em saúde no contexto dos processos de exclusão/inclusão social. Cad Saude Publica. 2003;19(6):1827-35. PMid:14999348. http://dx.doi.org/10.1590/ S0102-311X2003000600026.

8. Xavier IR. Projeto Migratório e Espaço: os migrantes bolivianos na Região Metropolitana de São Paulo [dissertação]. Campinas: Universidade Estadual de Campinas, Instituto de Filosofia e Ciências Humanas; 2010.

9. Silva ECC. Rompendo barreiras: os bolivianos e o acesso aos serviços de saúde na cidade de São Paulo. Travessia. Revista do Migrante. 2009;22(63):26-31. http://dx.doi.org/10.4322/dae.2014.028.

10. Waldman TC. Movimentos migratórios sob a perspectiva do direito à saúde: imigrantes bolivianas em São Paulo. Revista de Direito Sanitário. 2011;12(1):90-114. http://dx.doi.org/10.11606/issn.2316-9044.v12ilp90-114.

11. Martes ACB, Faleiros SM. Acesso dos imigrantes bolivianos aos serviços públicos de saúde na cidade de São Paulo. Saúde Soc. 2013;22(2):351-64. http://dx.doi.org/10.1590/S0104-12902013000200008.

12. Rufino C, Amorim SG. Imigração internacional e Gestão Pública da Saúde na Região Metropolitana de São Paulo (RMSP): uma contextualização a partir das Unidades Básicas de Saúde (UBS). In: Anais do XVIII Encontro Nacional de Estudos Populacionais; 2012 nov 19-23; Águas de Lindóia, BR. São Paulo: ABEP; 2012.

13. Aguiar ME, Mota AO. Programa saúde da família no bairro do bom Retiro, SP, Brasil: a comunicação entre bolivianos e trabalhadores de saúde. Interface. 2014;18(50):493-506. http://dx.doi.org/10.1590/1807-57622013.0040.

14. Goldberg A, Silveira C. Social inequality, access conditions to public health care and processes of care in bolivian immigrants in Buenos Aires and São Paulo: a comparative inquiry. Saude Soc. 2013;22(2):1-14.

15. Chubaci RYS, Merighi MAB. A comunicação no processo da hospitalização do imigrante japonês. Rev Latino-am Enfermagem. 2002;10(6):805-12. http://dx.doi.org/10.1590/S0104-11692002000600009.
16. Kang S, Razzouk D, Mari JJ, Shirakawa I. The mental health of Korean immigrants in São Paulo, Brazil. Cad Saude Publica. 2009;25(4):819-26. PMid:19347208. http://dx.doi.org/10.1590/S0102-311X2009000400013.

17. Nogueira VMR, Fagundes HS. A implementação do Programa SIS Fronteiras: perspectivas para a ampliação do direito à saúde aos usuários estrangeiros na Fronteira Arco Sul. Serv Soc \& Saúde. 2014;13(2):245-60.

18. Jimenez RP, Nogueira VMR. La construcción de los derechos sociales y los sistemas sanitarios: los desafíos de las fronteras. Rev Katál. 2009;12(1):50-8.

19. Giovanella L, Guimarães L, Nogueira VMR, Lobato LVC, Damancena GN. Saúde nas fronteiras: acesso e demandas de estrangeiros e brasileiros não residentes ao SUS nas cidades de fronteira com países do Mercosul na perspectiva dos secretários municipais de saúde. Cad Saude Publica. 2007;23(2 Supl):S251-S266.

20. Nogueira VMR, Dal KR, Fermiano S. A diversidade ética e política na garantia e fruição do direito à saúde nos municípios brasileiros da linha da fronteira do MERCOSUL. Cad Saude Publica. 2007;23(2 Supl):227236.

21. Brasil. Lei no 8.080, de 19 de setembro de 1990. Dispõe sobre as condições para a promoção, proteção e recuperação da saúde, a organização e o funcionamento dos serviços correspondentes e dá outras providências. Diário Oficial da União, Brasília, DF, 20 de setembro de 1990.

22. Oliveira AAS. Análise de consulta sobre o direito à assistência terapêutica de estrangeiro no âmbito do sistema único de saúde. In: Escola da AdvocaciaGeral da União. Publicações da Escola da AGU: temas de direito e saúde. Brasília: Advocacia-Geral da União; 2010 [citado em 2015 Mar 03]. p. 131-144. Disponível em: http://www.agu.gov.br/sistemas/site/TemplateImagemTexto. aspx ?idConteudo $=158795$ \&ordenacao $=1$ \&id_site $=7529$

Recebido em: Set. 15, 2016 Aprovado em: Fev. 15, 2017 